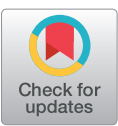

\section{Technical Note}

\title{
Endoport-assisted microsurgical treatment for a ruptured posterior cerebral artery aneurysm: A technical note
}

\author{
Juan Luis Gómez-Amador' ${ }^{1}$ Marcos Vinicius Sangrador-Deitos ${ }^{2}$, \\ Rodrigo Uribe-Pacheco ${ }^{2}$, Gerardo Yoshiaki Guinto-Nishimura², \\ Michel Gustavo Mondragón-Soto ${ }^{2}$ \\ ${ }^{7}$ Department of Skull-Base Neurosurgery, National Institute of Neurology and Neurosurgery \\ Manuel Velasco Sáurez, Mexico City, Mexico \\ ${ }^{2}$ Department of Neurosurgery, National Institute of Neurology and Neurosurgery Manuel \\ Velasco Sáurez, Mexico City, Mexico
}

\section{J Cerebrovasc Endovasc Neurosurg. 2022 March;24(1):73-78 \\ Received: 6 May 2021 \\ Revised: 9 August 2021 \\ Accepted: 13 August 2021}

Posterior Cerebral Artery aneurysms are scarce, yet its territory is frequently associated to large and giant aneurysms. Treatment is mostly a binary option between microsurgical clipping and endovascular coiling. Hybrid approaches are an option too, whereas innovation with less frequent techniques such as endoscope-controlled and endoscope-assisted procedure may provide a safer surgical approach with same successful results. Hereby we report a case of a 53 years old male examined at the ER after presenting generalized seizures and altered state of consciousness. Upon arrival, neurological evaluation revealed homonymous right hemianopia. Computed tomography (CT) scan revealed a subarachnoid hemorrhage and left parieto-occipital intraparenchymal hemorrhage with intraventricular extension; computed tomography angiogram (CTA) revealed an aneurysm at the left posterior cerebral artery (PCA) in its P4 segment. We performed a vascular exploration with drainage of the occipital and intraventricular hematoma through a single endoscopic port through transulcal approach guided by neuronavigation, in addition to clipping and aneurysmectomy. The combination of microsurgical clipping with previous Endoport-guided endoscopic procedure may be a surgical-operative option that not only may facilitate the approach to the desired lesion, but also provides a safer surgical scenario.

Keywords Aneurysm, Endoscopic neurosurgery, Aneurysm clipping, Endoport, Vascular neurosurgery, Technical note
This is an Open Access article distributed under the terms of the Creative Commons Attribution Non-Commercial License (http://creativecommons.org/licenses/ by-nc/4.0/) which permits unrestricted non-commercial use, distribution, and reproduction in any medium, provided the original work is properly cited.

\section{INTRODUCTION}

Posterior circulation aneurysms are infrequent, representing from 5 to $10 \%$ of intracerebral aneurysms. In 1966, the Cooperative Study of Intracranial Aneurysms 
and Subarachnoid Hemorrhage, reported a total of 2,672 single aneurysms, from which 144 (5.3\%) arose from the posterior or vertebro-basilar circulation, $22(15.2 \%)$ of these being from the posterior cerebral artery (PCA), for a total incidence of $0.8 \% .{ }^{10)}$ Solely, aneurysms of the PCA account for 0.7 to $2.3 \%$ of all intracranial aneurysms. ${ }^{7)}$ They may be treated through multiple approaches, either direct surgical clipping with a standard or minimally invasive procedure, or endovascular coil embolization. ${ }^{1)}$ Hereby we present this technical note about the endoscope-assisted microsurgical treatment of PCA aneurysm in a 53 years old male-patient.

\section{CASE REPORT}

A 53 years old male without any previous medical condition, arrived to the ER with his relative whom referred 3 hours of intense headache, somnolence, and disorientation right after witnessing sudden generalized seizures. At admission, general and neurological evaluation revealed Glasgow coma score (GCS) 13 (Ocular 3, Verbal 5, Motor 5), time and space disorientation, homonymous right hemianopia, and Kernig and Brudzinski signs present. Computed tomography (CT) scan was performed: simple sequences revealed a subarachnoid hemorrhage with left parieto-occipital intraparenchymal hemorrhage with extension to the lateral and third ventricles; computed tomography angiogram (CTA) revealed an aneurysm at the left PCA in its P4 segment (Fig. 1).

We performed a vascular exploration with drainage of the occipital and intraventricular hematoma through a single endoscopic port via a transulcal approach guided by neuronavigation, in addition to clipping and aneurysmectomy (Fig. 2). The patient was placed in a right park bench position with adequate padding of osseous surfaces. Guided with neuronavigation, a straight-line incision followed by a $6 \times 6 \mathrm{~cm}$ craniotomy were performed. Arachnoid dissection was completed over the interparietal sulcus and a $21 \mathrm{~mm} / 15 \mathrm{~mm} / 7$ $\mathrm{cm}$ TC model Vycor Viewsite Brain Access System (VBAS) (Vycor Medical ${ }^{\mathrm{TM}}$, FL, USA) was introduced directed towards the occipital horn of the left lateral ventricle until a parenchymal and ventricular hematoma consisting of organized clots was observed. A rigid straight $0^{\circ}$ endoscope held and fixed steady by the first assistant at all moments, guided the hematoma drainage. Meanwhile surgeon performs the microsurgical procedure with no need of a surgical microscope, drains the hematoma and subsequently performs subarachnoid dissection revealing a partially thrombosed aneurysm of the distal parieto-occipital artery of the left PCA. Proximal control was achieved with proper dissection and transient clipping of the proximal portion of P4. The aneurysm was permanently clipped with a $7 \mathrm{~mm}$
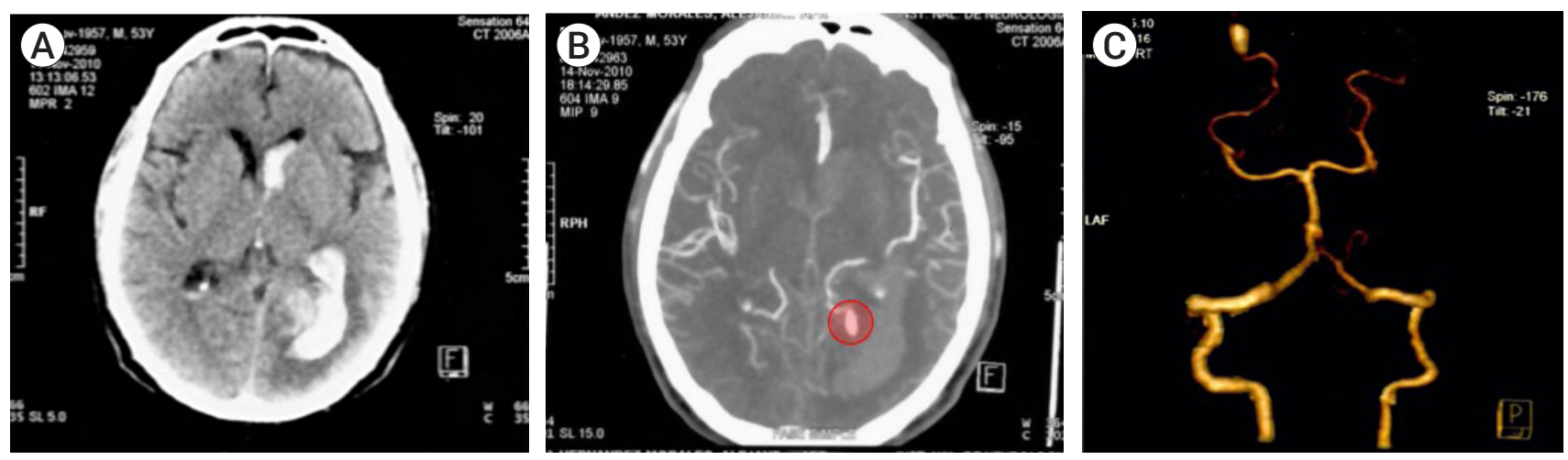

Fig. 1. (A) Preoperative axial CT scan showing subarachnoid hemorrhage with left parieto-occipital parenchymal hemorrhage with ventricular extension. No hydrocephalus nor midline deviation is observed. (B and C) Preoperative CTA: (B) Axial scan in which an irregular, hyperdense projection arising from the posterior circulation is observed (red circle), (C) 3D reconstruction shows a saccular aneurysm from the P4 segment of the left PCA. CT, computed tomography; CTA, computed tomography angiogram; PCA, posterior cerebral artery 

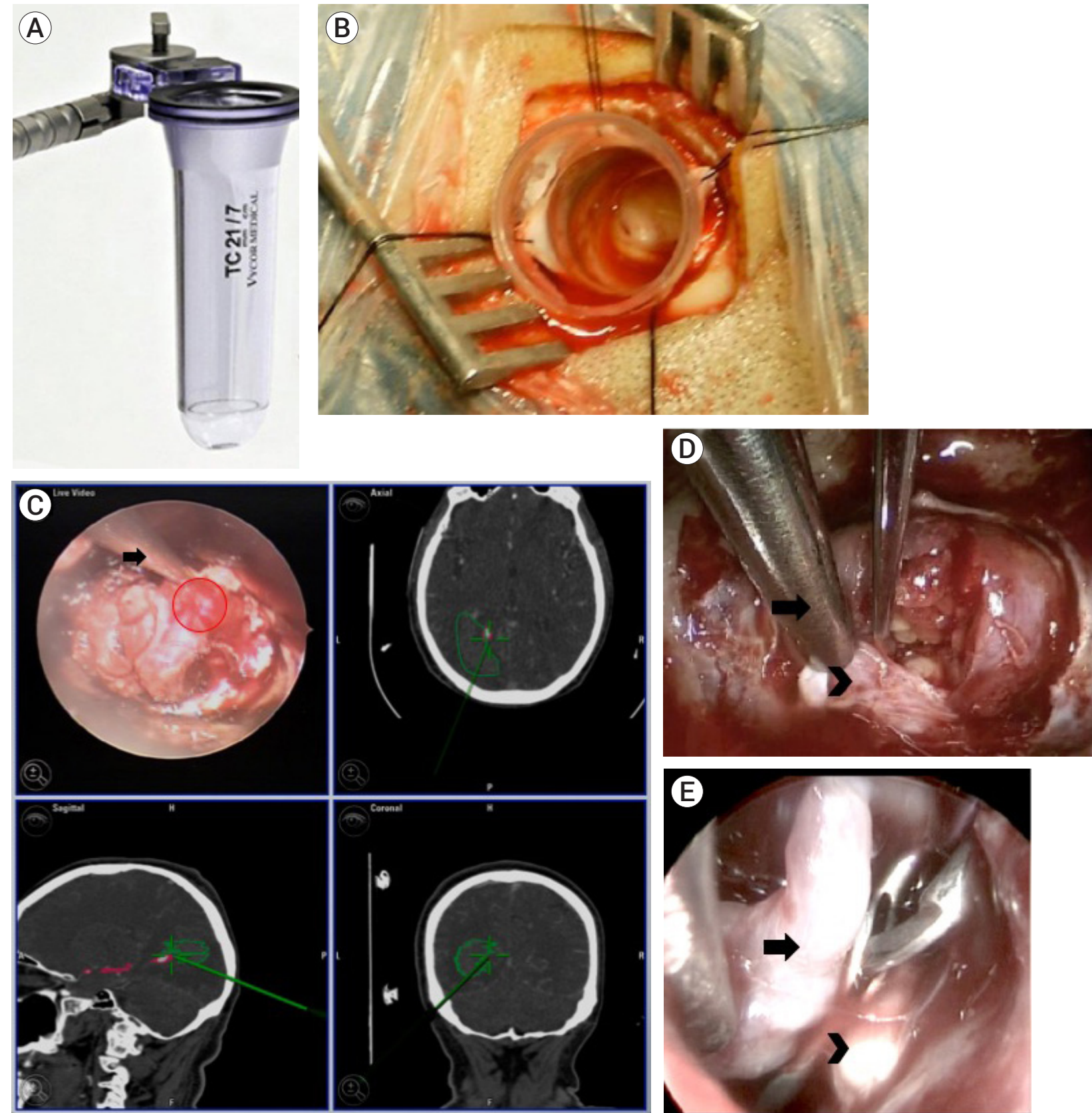

Fig. 2. Surgical approach and findings. (A) $21 \mathrm{~mm} / 15 \mathrm{~mm} / 7 \mathrm{~cm}$ TC model Vycor Viewsite Brain Access System (VBAS) (Vycor Medical $^{\mathrm{T} M}, \mathrm{FL}$, USA). (B) After performing a tailored incision and craniotomy, the endoport was introduced through the interparietal sulcus. (C) Upper left inset: endoscopic view after arachnoid dissection, in which the aneurysm dome and neck can be observed (red circle), signaled by the neuronavigation probe (arrow). Upper right inset: axial, lower left inset: sagittal, and lower right inset: coronal reconstructions in the neuronavigation system in which the navigation probe (green line), hematoma (green continuous line), and aneurysm (pink circle) can be observed. (D) Arachnoid dissection of the perianeurysmatic architecture in order to achieve an adequate visualization of the aneurysm's neck (arrowhead), at the tip of the suction probe (arrow). (E) Endoscopic view after aneurysm exclusion. A straight $7 \mathrm{~mm}$ clip was placed between the aneurysm's dome (arrowhead) and the P4 segment of the posterior cerebral artery (arrow).

straight clip, followed by dome resection. Closure of the approach was performed in a standard fashion. The patient's postoperative course was uneventful, with a sequel homonymous right hemianopia. Postoperative computed tomography showed absence of parenchymal or intraventricular hemorrhage, yet parenchymal 
hypodensity related to a left PCA infarction, which explained the clinical postchiasmatic sequel (Fig. 3).

\section{DISCUSSION}

The use of minimally invasive surgery (MIS) has proven to be effective in the management of aneurysms. Endoscope-assisted microneurosurgery for aneurysms was first reported in 1994 by Fischer and Mustafa, ${ }^{5)}$ where they emphasized the benefits of an improved visualization of anatomical structures around the aneurysm, allowing for better dissection and clipping technique. Yet the disadvantages these authors report are the difficulty to handle it, as well as the need to change from microscopical view to endoscopic - video monitoring view. ${ }^{5)}$ Perneczky declared that the use of an endoscope is specifically indicated for deep-seated lesions, especially aneurysms of the posterior circulation. He refers to a better control over aneurysm and adjacent nerves which leads to fewer intraoperative aneurysm ruptures and nerve injury comorbidity. ${ }^{14)}$ However, several studies have shown the usefulness of endoscopic view in most common aneurysm sites, not necessarily posterior circulation. ${ }^{19)}$ Some describe the main advantage of these techniques as a capability to "look around a corner", which provides a safer surgery. Deep-seated or intraventricular aneurysms, especially those surrounded by intracerebral or intraventricular hematomas, are the best candidates for minimally invasive endoscope / endoport-assisted surgery guided by neuronavigation. On the other hand, intracerebral hemorrhage (ICH), has become one of the most common indications for minimally invasive surgery. Conventional surgery for ICH evacuation has failed to prove any benefit over medical management, as demonstrated in the Surgical Trial in Intracerebral Hemorrhage (STICH) I and II, ${ }^{12) 13)}$ leaving MIS as a promising resource.

The PCA arises at the basilar bifurcation, encircles the brainstem passing through the crural and ambient cisterns, before reaching the quadrigeminal cistern, to finally distribute along the caudal pole of the hemisphere. According to different authors, it is divided either in 4 segments or in 6 sections. ${ }^{16)} \mathrm{P} 1$ and P2 segments which tally with section $\mathrm{C}$ (junction with the posterior communicating artery), are the most frequent
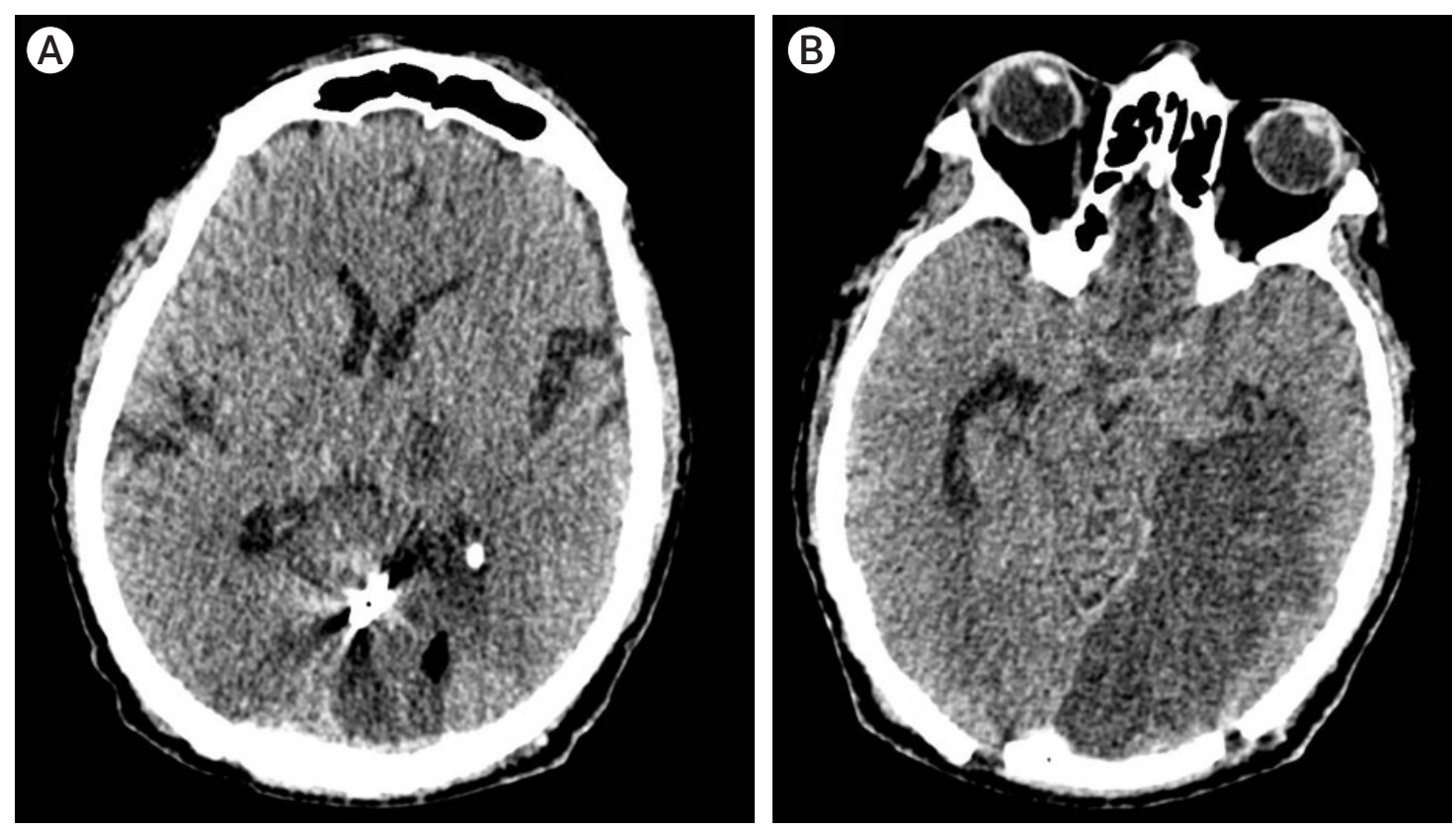

Fig. 3. Postoperative CT scan (A and B): axial reconstructions with no residual hemorrhage. (A) Aneurysm was trapped by clipping. (B) Left occipito-temporal hypodensity corresponding to an ipsilateral PCA remaining infarction. CT, computed tomography; PCA, posterior cerebral artery 
site for aneurysm formation within the PCA. ${ }^{46) 15)}$ There is a higher incidence of large and giant aneurysms in this site when compared to other anatomic locations (23\% of PCA aneurysms, versus 3-5\% in other locations). ${ }^{3) 11}$ Coexisting vascular lesions have been described as well: multiple aneurysms, arteriovenous malformations, and Moya-Moya disease have been reported. ${ }^{1)}$

Treatment of PCA aneurysms has several management options. Surgical clipping currently remains as the milestone treatment, where suitable transcranial approaches most widely used are pterional, subtemporal, and occipital interhemispheric, in order to reach and expose P1-P2 segments and corridors. ${ }^{18)}$ Endovascular management has increased, selective aneurysm occlusion with coils can be safely performed with good outcomes on PCA saccular aneurysms. Hybrid treatment as with endovascular coiling followed by neuroendoscopic removal of intraventricular clots have shown improvement in hydrocephalus and GRAEB score, however, overall outcome did not show such good results. ${ }^{11)}$

Endoscopic procedures in contemporary neurosurgery have become one of its finest workhorses, and has extended the neurosurgeon's equipment and surgical options. Hopf and Perneczky proposed a classification of endoscopic techniques: ${ }^{8177}$

1. Endoscopic neurosurgery: mainly used in ventricular endoscopy.

2. Endoscope-controlled microneurosurgery: endonasal endoscopic skull base surgery, endoport surgery, endoscopic transcranial surgery.

3. Endoscope-assisted microneurosurgery: use of the endoscope and microscope in the same operation.

In our case, we present a combined endoscope-controlled and endoscope-assisted procedure. Joining these endoscopic techniques, the use of a tubular sheath, so-called "endoport", guided by a neuronavigation system has expanded even more the armamentarium when facing deep-seated cerebral lesions. ${ }^{9)}$ Classically, in order to reach these lesions, much retraction had to be applied on the overlying cortex, with undesirable effects. The use of an endoport distributes the retraction forces evenly over the surface of the sheath. Multiple lesions can be managed through Endoport-guided endoscopic procedures: cavernomas, gliomas, cysts, abscesses, meningiomas, metastases, aneurysms, etc. ${ }^{2)(12)}$

\section{CONCLUSIONS}

Aneurysms of the posterior circulation have always represented a challenge for even the most experienced neurosurgeons. Nonetheless, the efficacy in aneurysm neurosurgery can be improved with the assistance of endoscopy, neuronavigation, and endoport, novel surgical technique useful for minimally invasive procedures.

\section{Disclosure}

The authors report no conflict of interest concerning the materials or methods used in this study or the findings specified in this paper.

\section{REFERENCES}

1. Ciceri EF, Klucznik RP, Grossman RG, Rose JE, Mawad ME. Aneurysms of the posterior cerebral artery: classification and endovascular treatment. AJNR Am J Neuroradiol. 2001 Jan;22(1):27-34.

2. Ding D, Starke RM, Crowley RW, Liu KC. Endoport-assisted microsurgical resection of cerebral cavernous malformations. J Clin Neurosci. 2015 Jun;22(6):1025-9.

3. Drake CG. Giant posterior cerebral aneurysms: 66 patients. in Drake CG, Peerless SJ, Hruesniemi JA (ed). Surgery of Vertebrobasilar Aneurysms: London, Ontario, Experience on 1767 Patients. New York: Springer Verlag, 1996. p. 230-48.

4. Ferrante L, Acqui M, Trillo G, Lunardi P, Fortuna A. Aneurysms of the posterior cerebral artery: do they present specific characteristics? Acta Neurochir (Wien). 1996;138(7):840-52.

5. Fischer J, Mustafa H. Endoscopic-guided clipping of cerebral aneurysms. Br J Neurosurg. 1994;8(5):559-65.

6. Gerber CJ, Neil-Dwyer G. A review of the management of 15 cases of aneurysms of the posterior cerebral artery. Br J Neurosurg. 1992;6(6):521-7.

7. Honda M, Tsutsumi K, Yokoyama H, Yonekura N, Nagata I. Aneurysms of the posterior cerebral artery: retrospective 
review of surgical treatment. Neurol Med Chir (Tokyo). 2004 Apr;44(4):164-8.

8. Hopf NJ, Perneczky A. Endoscopic neurosurgery and endoscope-assisted microneurosurgery for the treatment on intracranial cysts. Neurosurgery. 1998 Dec;43(6):1330-6.

9. Jo KW, Shin HJ, Nam DH, Lee JI, Park K, Kim JH, et al. Efficacy of endoport-guided endoscopic resection for deep-seated brain lesions. Neurosur Rev. 2011 Oct;34(4):457-63.

10. Locksley HB. Natural history of subarachnoid hemorrhage, intracranial aneurysms and arteriovenous malformations. Based on 6368 cases in the cooperative study. J Neurosurg. 1966 Aug;25(2):219-39.

11. Longatti P, Fiorindi A, Di Paola F, Curtolo S, Basaldella L, Martinuzzi A. Coiling and neuroendoscopy: a new perspective in the treatment of intraventricular haemorrhages due to bleeding aneurysms. J Neurol Neurosurg Psychiatry. 2006 Dec;77(12):1354-8.

12. Mendelow AD, Gregson BA, Fernandes HM, Murray GD, Teasdale GM, Hope DT, et al. STICH investigators. Early surgery versus initial conservative treatment in patients with spontaneous supratentorial intracerebral haematomas in the International Surgical Trial in Intracerebral Hemorrhage (STICH): a randomized trial. Lancet. 2005 Jan 29-Feb 4; 365(9457):387-97.
13. Mendelow AD, Gregson BA, Rowan EN, Murray GD, Gholkar A, Mitchell PM, et al. Early surgery versus initial conservative treatment in patients with spontaneous supratentorial lobar intracerebral haematomas (STICH II): a randomized trial. Lancet. 2013 Aug;382(9890):397-408.

14. Perneczky A, Boecher-Schwarz HG. Endoscope-assisted microsurgery for cerebral aneurysms. Neurol Med Chir (Tokyo). 1998;38(Suppl):33-4.

15. Pia HW, Fontana H. Aneurysms of the posterior cerebral artery. Locations and clinical pictures. Acta Neurochir (Wien). 1977;38(1-2):13-35.

16. Rhoton AL Jr. The supratentorial arteries. Neurosurgery. 2002 Oct;51(4 Suppl):S53-120.

17. Schroeder HWS. General principles and intraventricular neuroendoscopy: endoscopic techniques. World Neurosurg. 2013 Feb;79(2 Suppl):S14.e23-8.

18. Terasaka S, Sawamura Y, Kamiyama H, Fukushima T. Surgical approaches for the treatment of aneurysms on the P2 segment of the posterior cerebral artery. Neurosurgery. 2000 Aug;47(2):359-64; discussion 364-6.

19. Wong J, Tymianski R, Radovanovic I, Tymianski M. Minimally invasive surgery for cerebral aneurysms. Stroke. 2015 Sep;46(9): 2699-706. 